\title{
Arabidopsis thaliana alternative dehydrogenases: a potential therapy for mitochondrial complex I deficiency? Perspectives and pitfalls
}

\author{
Alessia Catania ${ }^{1,2+}$ D. Arcangela luso ${ }^{3,4+}$, Juliette Bouchereau ${ }^{1,5+}$, Laura S. Kremer ${ }^{3,4}$, Marina Paviolo ${ }^{1,5}$, \\ Caterina Terrile ${ }^{3}$, Paule Bénit ${ }^{1}$, Allan G. Rasmusson ${ }^{6}$, Thomas Schwarzmayr ${ }^{3,7}$, Valeria Tiranti ${ }^{2}$, Pierre Rustin ${ }^{1 \dagger}$, \\ Malgorzata Rak ${ }^{1 \dagger}$, Holger Prokisch ${ }^{3,4^{*}+}$ (D) and Manuel Schiff ${ }^{1,5+}$
}

\begin{abstract}
Background: Complex I (Cl or NADH:ubiquinone oxidoreductase) deficiency is the most frequent cause of mitochondrial respiratory chain defect. Successful attempts to rescue $\mathrm{Cl}$ function by introducing an exogenous NADH dehydrogenase, such as the NDI1 from Saccharomyces cerevisiae (ScNDI1), have been reported although with drawbacks related to competition with $\mathrm{Cl}$. In contrast to ScNDI1, which is permanently active in yeast naturally devoid of $\mathrm{Cl}$, plant alternative $\mathrm{NADH}$ dehydrogenases $(\mathrm{NDH}-2)$ support the oxidation of $\mathrm{NADH}$ only when the $\mathrm{Cl}$ is metabolically inactive and conceivably when the concentration of matrix NADH exceeds a certain threshold. We therefore explored the feasibility of $\mathrm{Cl}$ rescue by $\mathrm{NDH}-2$ from Arabidopsis thaliana (At) in human $\mathrm{Cl}$ defective fibroblasts.

Results: We showed that, other than ScNDI1, two different NDH-2 (AtNDA2 and AtNDB4) targeted to the mitochondria were able to rescue $\mathrm{Cl}$ deficiency and decrease oxidative stress as indicated by a normalization of SOD activity in human Cl-defective fibroblasts. We further demonstrated that when expressed in human control fibroblasts, AtNDA2 shows an affinity for NADH oxidation similar to that of $\mathrm{Cl}$, thus competing with $\mathrm{Cl}$ for the oxidation of $\mathrm{NADH}$ as opposed to our initial hypothesis. This competition reduced the amount of ATP produced per oxygen atom reduced to water by half in control cells.

Conclusions: In conclusion, despite their promising potential to rescue $\mathrm{Cl}$ defects, due to a possible competition with remaining $\mathrm{Cl}$ activity, plant $\mathrm{NDH}-2$ should be regarded with caution as potential therapeutic tools for human mitochondrial diseases.
\end{abstract}

Keywords: Mitochondria, Mitochondrial diseases, Complex I, Alternative dehydrogenases, Arabidopsis thaliana, AtNDA2

\section{Introduction}

Human NADH:ubiquinone oxidoreductase or complex I (CI) is the largest complex of the respiratory chain, with

\footnotetext{
* Correspondence: prokisch@helmholtz-muenchen.de

${ }^{+}$Alessia Catania, Arcangela luso, Juliette Bouchereau, Pierre Rustin,

Malgorzata Rak, Holger Prokisch and Manuel Schiff contributed equally to this work.

${ }^{3}$ Institute of Human Genetics, Helmholtz Zentrum München, German Research Center for Environmental Health, Neuherberg, Germany ${ }^{4}$ Institute of Human Genetics, Technische Universität München, Munich, Germany

Full list of author information is available at the end of the article
}

a mass of $980 \mathrm{kDa}$ and 44 different subunits encoded by both mitochondrial and nuclear genomes [1].

CI catalyzes the consecutive transfer of two electrons, one per time, to a ubiquinone pool for each molecule of $\mathrm{NADH}$ oxidized. $\mathrm{NADH}$ oxidizing activity of $\mathrm{CI}$ is tightly controlling intra-mitochondrial metabolism, and electron transfer is coupled to both heat and ATP generation. Electron transfer is associated with the pumping of $4 \mathrm{H}+$ across the inner mitochondrial membrane, which sustains part of the mitochondrial membrane potential [2]. The 44 subunits are arranged in three functional modules: the $\mathrm{N}$

(c) The Author(s). 2019 Open Access This article is distributed under the terms of the Creative Commons Attribution 4.0 International License (http://creativecommons.org/licenses/by/4.0/), which permits unrestricted use, distribution, and 
module involved in oxidizing $\mathrm{NADH}$, the $\mathrm{Q}$ module involved in reducing ubiquinone, and the $\mathrm{P}$ module dedicating proton translocation [3]. A number of mutations in nuclear and in mitochondrial genes coding for many of the 44 subunits, as well as in genes coding for assembly or regulatory factors, have been shown to result in CI deficiency [4]. Therefore, CI deficiency can result in a combination of abnormalities: impaired oxidation of NADH to $\mathrm{NAD}+$, which alters the NADH/NAD+ ratio and leads to intra-mitochondrial metabolic disequilibrium and ultimately to lactic accumulation, release of electrons that are not correctly channeled to the ubiquinone subsequently generating radical oxygen species (ROS), and loss of proton pumping activity, which reduces mitochondrial potential, hence lowering ATP synthesis.

In microbes, fungi, plants, and also in some metazoan phyla (but not in arthropods or vertebrates), two key steps of the mitochondrial respiratory chain, namely ubiquinone reduction and ubiquinol oxidation, differ from mammals as they involve bypassing enzymes: alternative NADH dehydrogenases (NDH-2) and alternative oxidases (AOXs). $\mathrm{NDH}-2$ can functionally replace $\mathrm{NADH}$ oxidizing activity of $\mathrm{CI}$, transferring electrons from NADH directly to ubiquinone, while AOXs can be a functional substitute of complexes III and IV (AOXs being able to transfer electrons from a ubiquinol pool directly to oxygen, see Fig. 1) [5].

These alternative enzymes possess some key properties that distinguish them from other mitochondrial complexes: they are single or oligo subunit, non-proton pumping enzymes, as the energy they convey during their activation does not support mitochondrial potential; they are not inhibited by cytochrome pathway inhibitors (e.g. rotenone and cyanide) and, in contrast to other mitochondrial complexes, they are not transmembrane proteins but are associated to either the inner or the outer surface of the inner mitochondrial membrane [6, 7].

In Saccharomyces cerevisiae CI is absent and replaced by the ScNDI1 protein. In an attempt to rescue CI deficiency, Yagi and collaborators introduced this type II $\mathrm{NAD}(\mathrm{P}) \mathrm{H}$ dehydrogenase from yeast, ScNDI1, into mammalian cells with impaired CI. This resulted in the recovery of $\mathrm{NADH}$ oxidation and reduction of ROS production in a variety of CI defective cell cultures harbouring mutations in either ND4, ND5 or NDUFA1 $[8,9]$. Cells with $\mathrm{CI}$ deficiency acquired the ability to grow in a non-fermentable medium, such as galactose, upon transfection with ScNDI1. Moreover, ScNDI1 has proven beneficial in fly models of CI deficiency [10].

This concept was further developed in gene therapy approaches in mice and rats. Bypassing CI by expression of ScNDI1 was demonstrated to be well tolerated. Furthermore, ScNDI1 protected rat neurons against the CI specific inhibitor rotenone, rescued CI deficiency [11-13], and showed potential therapeutic effects in a mouse model of Parkinson disease [14].

However, when introduced into control HEK293 cells, ScNDI1 caused a decrease in the amount of ATP produced per oxygen reduced ( $\mathrm{P} / \mathrm{O}$ ratio) for the $\mathrm{CI}$-dependent respiration from a value of 2.5 to 1.8 [15], showing that ScNDI1 is active even in the presence of a fully functional $\mathrm{CI}$, therefore competing with $\mathrm{CI}$ for the oxidation of $\mathrm{NADH}$. Such competition could compromise energy production and lower mitochondrial potential, thus potentially leading to unpredictable metabolic consequences.

Unlike Saccharomyces cerevisiae, which lacks CI, many plants have NDH-2, which naturally coexist with CI. They oxidize NADH only in specific physiologic conditions, depending on the nature of the available organic

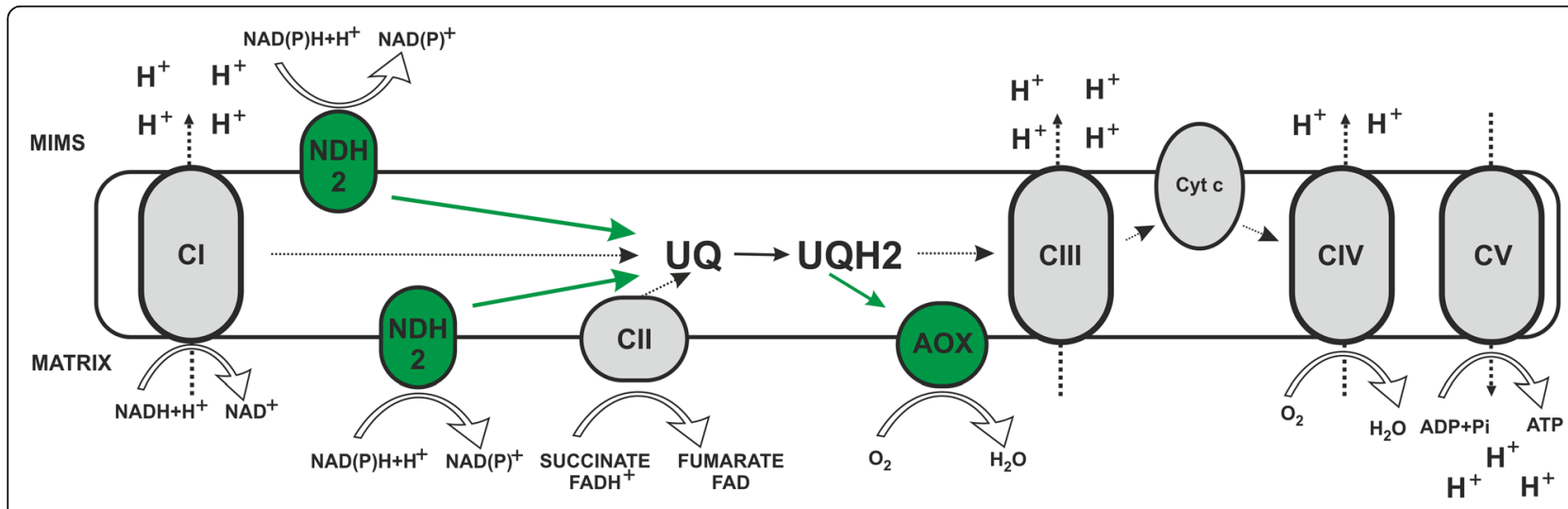

Fig. 1 Mitochondrial respiratory chain and alternative enzymes. Schematic representation of the canonical mitochondrial respiratory chain (in black and white) characterized by four multi-subunit complexes (Complex I, Complex II, Complex III and Complex IV) and two intermediary substrates (ubiquinone and cytochrome c) generating an electrochemical gradient through the inner mitochondrial membrane. Protons flow back to the matrix via Complex $V$ to produce ATP. The figure also illustrates alternative pathways of $\mathrm{NAD}(\mathrm{P}) \mathrm{H}$ and ubiquinol oxidation (in green) represented by alternative dehydrogenases $(\mathrm{NDH} 2)$ and alternative oxidases $(\mathrm{AOX})$, respectively. $\mathrm{Cl}$ to $\mathrm{CV}$, complexes I to $\mathrm{V}$; $\mathrm{UQ}$, ubiquinone; UQH2, ubiquinol; Cyt c, cytochrome c; MIMS, mitochondrial intermembrane space 
acids, considering that some alternative dehydrogenases from plants were shown to have a 3 to 10 folds higher $K_{M}$ for NADH than plant CI in native conditions [16-18], or hypothetically on the presence of specific matrix compartmentalized NADH pools.

Arabidopsis thaliana, in particular, expresses different isoforms of NDH-2 associated either to the inner or to the outer mitochondrial membrane. The intrinsic role of these alternative systems could be to maintain a redox balance and a proper turn-over of mitochondrial metabolism, continuing to oxidize substrates when the metabolic demand is modified. This particularly manifests during daylight exposition of plants, when OXPHOS is inhibited by the extensive mobilization of cytosolic ADP by the photosynthetic process: and indeed, it was shown that activation/expression of $\mathrm{NDH}-2$ takes place in physiologic conditions lowering CI activity [19].

Thus, the plant enzyme is expected to naturally take over for $\mathrm{NADH}$ oxidation only when $\mathrm{CI}$ is prevented from working, providing a potential mechanism to mitigate the redox imbalance in cells with defective $\mathrm{CI}$, without competing with its endogenous residual activity.

A very similar strategy based on the expression of the tunicate Ciona intestinalis alternative oxidase (AOX) has previously been shown to exert beneficial effects in counteracting the consequences of complexes III or IV respiratory chain deficiency in human cells and animal models [20], even though relevant constraints deriving from a profound influence on energy production and other biological processes have been recently reported after transfection in Drosophila [5].

Taken together, all these considerations open a way to forecast xenotopic transfection of genes encoding for plant's NDH-2 as a conceivable treatment for CI deficiency, as these enzymes should be active only when electron transfer from $\mathrm{NADH}$ through $\mathrm{CI}$ is impaired. Therefore, we evaluated the potential benefit of introducing alternative dehydrogenases AtNDA2 and AtNDB4 from Arabidopsis thaliana into a CI defective patient fibroblast cell line carrying a homozygous mutation in NDUFS4 and compared it to ScNDI1 from Saccharomyces cerevisiae. Moreover, we assessed kinetic and biochemical effects of one of these proteins (AtNDA2) in control fibroblasts.

\section{Materials and methods}

\section{Cell transfection and selection}

For the evaluation of the above-described therapeutic strategy on cellular models, we focused on control and CI defective human fibroblasts.

The control fibroblasts (NDHF) were purchased from Lonza (Cat. No. CC-2509). Patients' fibroblasts were obtained from skin biopsies of patients with signed informed consent. The CI defective cell line (79787) belongs to a patient affected with Leigh syndrome carrying the homozygous frameshift mutation c.462delA (p.Lys154fs) within NDUFS4, located in $5 \mathrm{q} 11$ and encoding for a CI subunit close to the catalytic region of NADH-quinone oxidoreductase. The mutation is predicted to result in the synthesis of a truncated protein. Indeed, the absence of NDUFS4 protein was previously reported in fibroblasts derived from patients harbouring the same NDUFS4 homozygous mutation [21].

Skin fibroblast cells were grown in Dulbecco's modified Eagle's medium (DMEM) with Glutamax +/- $4.5 \mathrm{~g} / \mathrm{L}$ Glucose, supplemented with $10 \%$ fetal calf serum (FBS), $2.5 \mathrm{mM}$ Pyruvate and maintained in a $5 \% \mathrm{CO} 2$ incubator at $37^{\circ} \mathrm{C}$. Patients' fibroblasts were obtained from skin biopsies of patients and signed informed consent. Selective growth of transfected cells was maintained by adding blasticidin $5 \mu \mathrm{g} / \mathrm{ml}$ to DMEM.

Control and patient fibroblasts were transfected with constructs containing the four NDH-2 genes of interest (AtNDA1, AtNDA2, AtNDB4 and ScNDI1) fused with human mitochondrial targeting signal (MTS) and a blasticidin resistance sequence (Additional file 1: Supplementary Methods). Transfection has been performed using a lentiviral vector from Invitrogen ${ }^{\mathrm{Tm}}$ (ViraPower $^{\mathrm{Tm}}$ HiPerform ${ }^{\mathrm{Tn}}$ ) according to Kremer and Prokisch [22]. Assessment of transduction efficacy and selection of transfected cell lines were performed using the results of qPCR (not shown) and oxygen consumption analysis (Fig. 2) as previously described [22].

\section{Enzymatic activity assay and determination of kinetic parameters}

Collection and permeabilization of fibroblasts were performed as previously described [23].

Spectrophotometric analysis of NADH:quinone oxidoreductase specific activity was performed on a Cary 60 spectrophotometer equipped with a18-cell holder maintained at $37^{\circ} \mathrm{C}$.

Measures of NADH:quinone oxidoreductase specific activity were performed in buffer A containing 10 $\mathrm{mM} \mathrm{KH_{2 }} \mathrm{PO}_{4}, \mathrm{pH} 7.2$ and $1 \mathrm{mg} / \mathrm{mL}$ BSA at wavelengths of $340 \mathrm{~nm}-380 \mathrm{~nm}$ to assess NADH oxidation using an extinction coefficient of 4.87 as previously described [23, 24].

The sample compartment was kept open in order to allow manual stirring of the cuvette content after each addition. For $K_{M}$ determination, samples $(8-20 \mu \mathrm{L})$ were added to water, incubated for $1 \mathrm{~min}$ before mixing with buffer A. Rotenone $(8 \mu \mathrm{M}), \mathrm{KCN}(650 \mu \mathrm{M})$, DCQ $(50 \mu \mathrm{M})$ were sequentially added to the cuvettes before starting the reaction with the substrate $\mathrm{NADH}$ (at concentrations ranging from 0.3 to $150 \mu \mathrm{M}$ ) and following the reaction kinetics. A comparative assay was performed without rotenone in order to quantify the amount of rotenone 


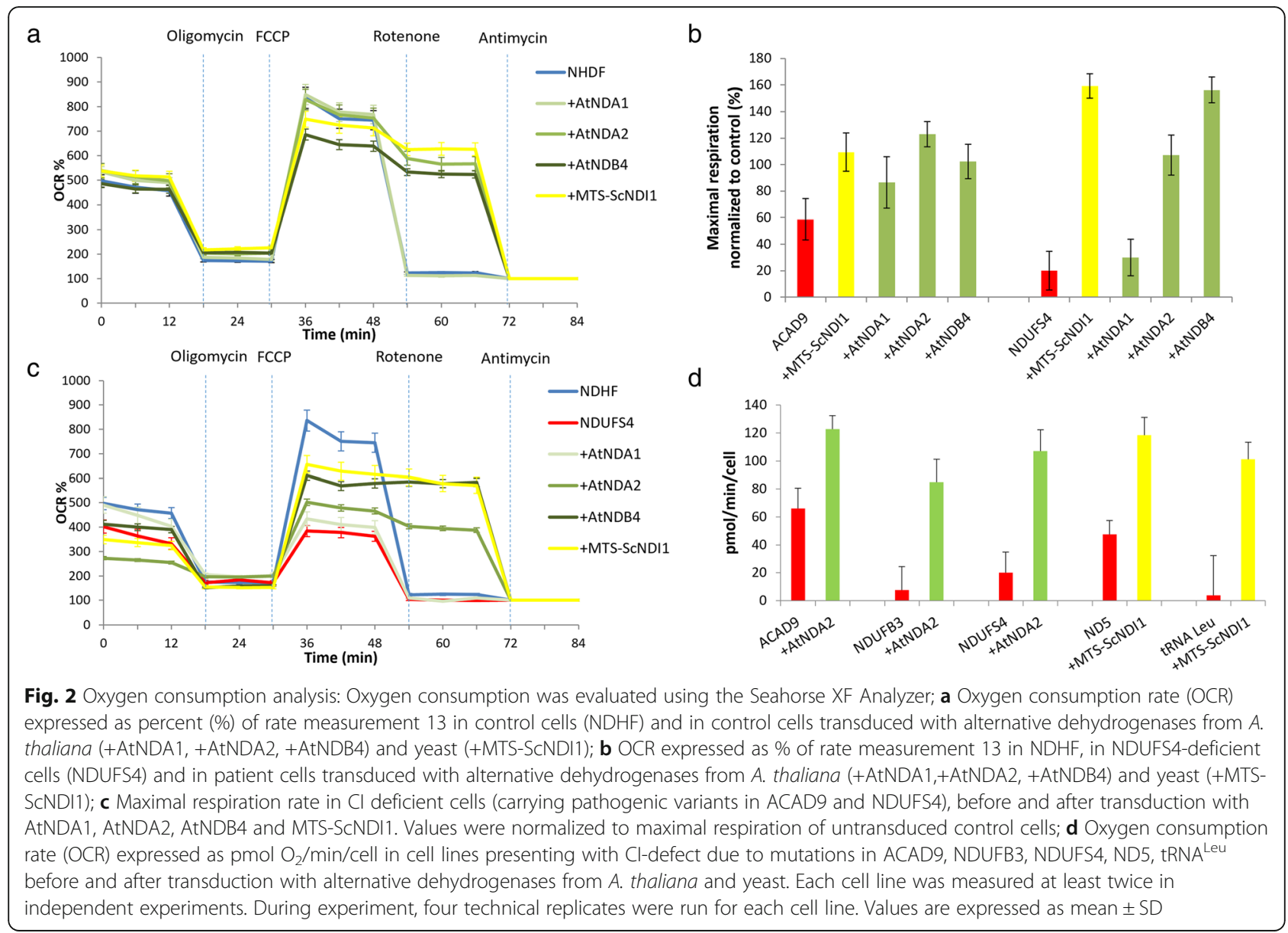

resistant NADH:quinone oxidoreductase activity. All the measurements were performed at least in triplicates.

$\mathrm{K}_{\mathrm{M}}$ and Vmax were estimated using an online available tool (http://www.ic50.tk/ $\mathrm{K}_{\mathrm{M}}$ vmax.html) using the MichaelisMenten model.

Proteins were measured according to Bradford [25].

\section{Superoxide dismutase (SOD) activity assessment}

SOD activity was measured according to Stefan L. Marklund following the described method of pyrogallol auto-oxidation inhibition. One unit of SOD inhibits 50\% of pyrogallol autoxidation, measured at $420 \mathrm{~nm}$ [26].

\section{$\mathrm{P} / \mathrm{O}$ assay}

Subconfluent fibroblasts $\left(75 \mathrm{~cm}^{2}\right.$ flask $)$ were trypsinized and the pellet was washed once with $1 \mathrm{~mL}$ PBS. The oxygen uptake was measured with an optic fiber equipped with an oxygen-sensitive fluorescent terminal sensor (Optode device: FireSting $\mathrm{O}_{2}$, Bionef, Paris, France). The optic fiber was fitted to a printed cap ensuring the closure of the quartz-cell yet allowing micro-injections $(0.6 \mathrm{~mm}$ hole diameter $)$ for concurrent measurement of oxygen uptake with mitochondrial potential (determined by the fluorescence change of 100 $\mathrm{nM}$ rhodamine). Cells were added to $750 \mu \mathrm{L}$ of buffer consisting of $0.25 \mathrm{M}$ sucrose, $15 \mathrm{mM} \mathrm{KCl}, 30 \mathrm{mM}$ $\mathrm{KH}_{2} \mathrm{PO}_{4}, 5 \mathrm{mM} \mathrm{MgCl}, 1 \mathrm{mM}$ EGTA, pH 7.4, followed by the addition of rhodamine $(100 \mathrm{nM})$, BSA $1 \mathrm{mg} / \mathrm{ml}$ and $0.01 \% \mathrm{w} / \mathrm{v}$ digitonin. The permeabilized cells were successively added followed by the addition of mitochondrial substrates $(6.25 \mathrm{mM}$ glutamate/malate or 6.25 $\mathrm{mM}$ succinate) and two consecutive injections of ADP ( $40 \mathrm{nmol}$ each) to ensure state 3 (phosphorylating) conditions or ATP $(40 \mathrm{nmol})$ in order to estimate ATP recycling due to ATPases activity. The reaction was followed until state 4 (the respiratory rate after all the ADP has been phosphorylated to form ATP) was reached back and maintained. Respiratory rates during state 3 and state 4 were estimated as the speed of oxygen consumption ( $\mathrm{nmol} / \mathrm{min}$ ) adjusted to protein concentration $(\mu \mathrm{g})$ in each cuvette. Respiratory control index was later calculated as the ratio between state 3 to state 4 respiratory rates. $\mathrm{P} / \mathrm{O}$ values (corresponding to the number of ATP molecules produced for every oxygen atom consumed) were also measured as the ratio between concentration (in nmol) of ADP (or ATP) added 
to the cuvette and the amount of oxygen atoms (nmol of molecular oxygen ${ }^{*} 2$ ) consumed during state 3 to state 4 transition. All the assays were repeated at least three times. Protein content was measured according to Bradford [25].

\section{RNA sequencing}

RNA sequencing was performed as described [27]. Briefly, RNA was isolated from whole-cell lysates using the AllPrep RNA Kit (Qiagen) and RNA integrity number (RIN) was determined with the Agilent 2100 BioaAnalyzer (RNA 6000 Nano Kit, Agilent). For library preparation, $1 \mu \mathrm{g}$ of RNA was poly(A)- selected, fragmented and reverse transcribed with the Elute, Prime and Fragment Mix (Illumina). End repair, Atailing, adaptor ligation and library enrichment were performed as described in the Low Throughput protocol of the TruSeq Stranded mRNA Sample Prep Guide (Illumina). RNAcDNA libraries were assessed for quality and quantity with the Agilent 2100 BioaAnalyzer and quantity using the Quant-iT PicoGreen dsDNA Assay Kit (Life Technologies). RNA libraries were sequenced as $150 \mathrm{bp}$ paired-end runs on an Illumina HiSeq4000 platform. The STAR aligner* (v 2.4.2a) with modified parameter settings (--twopassMode $=$ Basic) was used for split-read alignment against the human genome assembly hg19 (GRCh37) and UCSC knownGene annotation. Prior alignment, the reference genome sequence was augmented by two new contigs, one for each plant gene (NDA2 and NDB4, respectively). The nucleotide sequences of these two genes corresponded to the transgenic constructs cloned in the lentiviral vector (see Additional file 1: Supplementary Methods). To quantify the number of reads mapping to annotated genes we used HTseq-count (v0.6.0). FPKM (Fragments Per Kilobase of transcript per Million fragments mapped) values were calculated using custom scripts.

\section{Statistical analysis}

All data are expressed as the mean $\pm \mathrm{SD}$, and comparisons between groups performed using Student's $t$ test.

\section{Results \\ Proof of concept that NDH-2 dehydrogenases counteract $\mathrm{Cl}$ deficiency}

Preliminary assays on different CI defective fibroblast cell lines had showed the ability of several NDH-2 to rescue respiration defect (Fig. $2 \mathrm{~b}-\mathrm{d}$ ). We decided to focus our subsequent analysis on three NDH-2: ScNDI1, the internal NDH-2 of Saccharomyces cerevisiae; AtNDB4, an Arabidopsis thaliana NDH-2 localized to the external side of inner mitochondrial membrane (IMM); AtNDA2, another Arabidopsis thaliana $\mathrm{NDH}-2$ localized to the internal side of IMM.

As expected, transfection of control fibroblasts with AtNDA2, AtNDB4, and ScNDI1 led to rotenoneresistance without any significant effect on overall respiration rate (Fig. 2a). In order to examine the rescuing efficiency of plant NDH-2 in more depth, we chose to focus on fibroblasts carrying a pathogenic homozygous mutation in the nuclear gene NDUFS4, as a wellestablished cellular model of complex I deficiency. Indeed, consequences of deleterious mutations affecting NDUFS4 have been thoroughly studied on several patient cell lines and on whole-body and tissue specific knockout mice [28].

Therefore, we verified and confirmed that besides conferring rotenone resistance, all the aforementioned NDH-2 (ScNDI1, AtNDA2 and AtNDB4) were able to restore respiration when expressed in NDUFS4 deficient fibroblasts, nearly reaching control levels. (Fig. 2c).

\section{Expression of NDH-2 dehydrogenases does not affect growth of cultured human fibroblasts}

ScNDI1, AtNDA2, and AtNDB4 transfected control fibroblasts (NHDF) and CI-defective fibroblasts (NDUFS4) exhibited comparable growth rates when compared to the corresponding untransfected control, both in glucose $(4.5 \mathrm{~g} / \mathrm{L})$ and glucose-deprived media (not shown).

\section{At-NDA2 and at-NDB4 rescue NADH:quinone oxidoreductase activity of NDUFS4 mutant fibroblasts} We further confirmed the observed rescue by measuring NADH:quinone oxidoreductase specific activity by spectrophotometry in control cells and NDUFS4 mutated fibroblasts before and after transfection by ScNDI1, AtNDA2, and AtNDB4, show that all three dehydrogenases were able to rescue the $\mathrm{CI}$ defect (Table 1). We could also observe that, while AtNDA2 and AtNDB4 restored CI activity to levels comparable to those observed in control cells, ScNDI1 transfected cells exhibited levels of NADH:quinone oxidoreductase activity much higher than untransfected cells (Table 1).

\section{SOD activity in NDUFS4 deficient cells}

As a direct effect of defective CI activity and consequent increase of ROS production, SOD activity is shown to be significantly higher in NDUFS4 mutated patient's cell (Fig. 3). Transfection with AtNDA2 and AtNDB4 but not with ScNDI1 was almost able to decrease SOD activity to levels observed in control fibroblasts (Fig. 3). 
Table $1 \mathrm{NADH}$ :quinone oxidoreductase activity in control and transfected cells

\begin{tabular}{llll}
\hline Cell line & $\begin{array}{l}\text { NADH:quinone oxidoreductase } \\
\text { specific activity w/o rotenone } \\
\text { (nmol/min/mg Prot) }\end{array}$ & $\begin{array}{l}\text { NADH:quinone oxidoreductase } \\
\text { specific activity with rotenone } \\
\text { (nmol/min/mg Prot) }\end{array}$ & $\begin{array}{l}\text { Inhibition } \\
(\%)\end{array}$ \\
\hline NHDF & $7.7( \pm 0.5)$ & $1.7( \pm 0.2)$ & $78.3 \%$ \\
79,787 & $2.5( \pm 0.2)$ & $1.4( \pm 0.1)$ & $42.6 \%$ \\
$79,787-T-S C N D 11$ & $24.0( \pm 8.7)$ & $20.7( \pm 8.3)$ & $13.7 \%$ \\
$79,787-T-A t N D B 4$ & $11.8( \pm 2.4)$ & $10.6( \pm 1.9)$ & $10.0 \%$ \\
$79,787-T-A T N D A 2$ & $5.3( \pm 0.9)$ & $5.3( \pm 0.9)$ & $2.7 \%$ \\
\hline
\end{tabular}

Spectrophotometric assessment of NADH:quinone oxidoreductase specific activity in control cells (NDHF) and NDUFS4 cell line untransfected (79787) and transfected with alternative NADH oxidases (ScNDI1, AtNDA2 and AtNDB4). Values are expressed as mean \pm SD

\section{AtNDA2 and AtNDB4 expression in control cell lines}

We evaluated by RNA sequencing the expression levels of CI subunits, AtNDA2, and AtNDB4 in control cells before and after the stable transduction with the plant genes AtNDA2 and AtNDB4 (RNA sequencing was not performed on NDUFS4 deficient fibroblasts due to scarcity of material). We evaluated the FPKM values for CI subunits before and after transduction. The median FPKM of CI subunits was similar in all cell lines, indicating that the transduction with the plant genes did not affect the expression level of CI subunits (median FPKM between 30 and 35, Table 2). AtNDA2 had an expression level of 25 FPKM, which falls in the range of CI subunit expression, while AtNDB4 had an expression level of 127 FPKM, much higher than the median expression level of CI subunits (Table 2). In A. thaliana the endogenous expression of NDA2 and NDB4 is significantly lower than the expression of CI subunits, in all

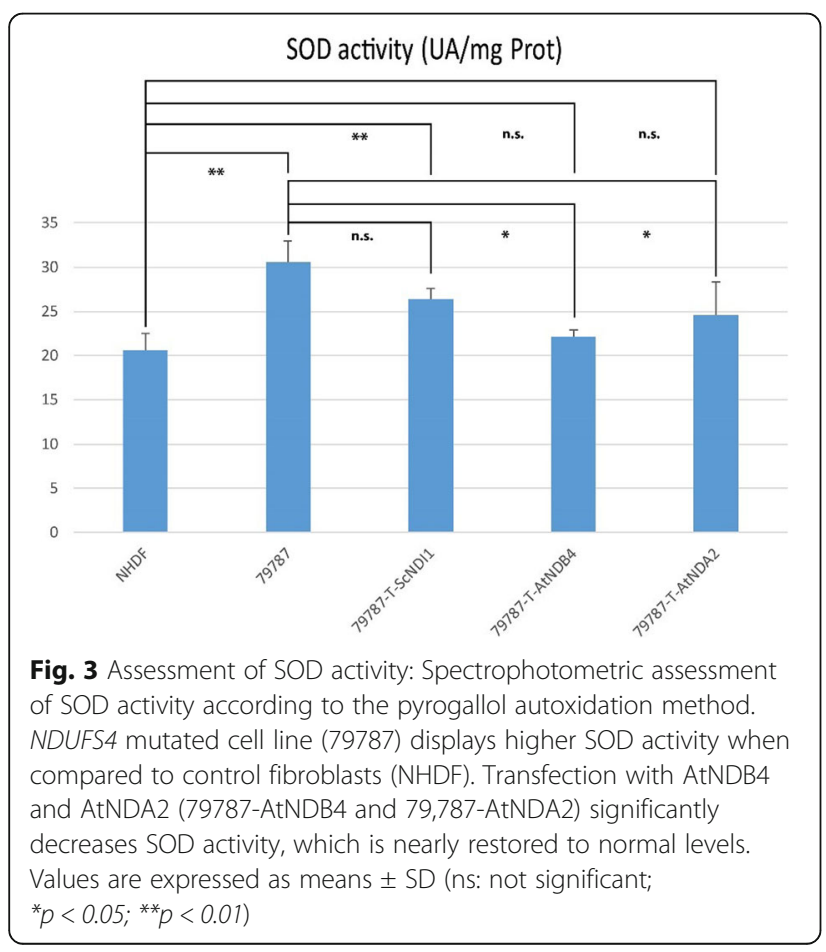

parts of the plant (flower, root, leaf, and fruit). The expression of NDA2 is 10 times lower than the median expression of CI subunits, while NDB4 is almost 500 times lower than the median expression of CI subunits [29] (Additional file 1: Table S1).

\section{AtNDA2 competes with $\mathrm{Cl}$ when expressed in human fibroblasts}

Thereafter, in view of its expression profile in A. thaliana (Add file 1), we selected the AtNDA2 to verify the lack of competition between $\mathrm{CI}$ and plant NDH-2 when expressed in control fibroblasts. We first studied the P/ $\mathrm{O}$ ratio with different substrates, the latter being supposedly decreased if $\mathrm{NADH}$, normally oxidized by the proton-motive $\mathrm{CI}$, is diverted to AtNDA2. $\mathrm{P} / \mathrm{O}$ calculation is usually performed on isolated mitochondria in order to eliminate cytosolic ATPases activity. ATPases increase ADP recycling, allowing for a continuous stimulation of the mitochondrial ATP synthase and respiration, thus affecting state 4 establishment. However, taking into consideration the scarcity of material and the slow growth rate of fibroblasts, we performed the assays using permeabilized cells. As expected, the observed P/ $\mathrm{O}$ values were underestimated comparing to the ones measured on purified mitochondria (about 2.5 for NADH related substrates and 1.5 for succinate, respectively)-see Hinkle et al. [30] for a complete review on this topic. Nevertheless, using this approach, we were able to measure the $\mathrm{P} / \mathrm{O}$ ratio (Fig. 4). Unexpectedly, we evidenced a competition between AtNDA2 and functional mitochondrial respiratory chain CI during glutamate/ malate oxidation in the control cell line expressing AtNDA2 (Fig. 4). Transfected cells showed $\mathrm{P} / \mathrm{O}$ values lowered by half $(0.43 \pm 0.08)$ in comparison to untransfected cells $(0.9 \pm 0.1)$. Moreover, respiratory control index, calculated as the ratio between state 3 and state 4 , which represents a numeric estimation of mitochondrial coupling efficiency, was also clearly lowered in transfected cells under glutamate/malate stimulation (Fig. 5).

A further validation of these results came from $\mathrm{P} / \mathrm{O}$ data obtained with succinate, the substrate of CII. Indeed, although $\mathrm{P} / \mathrm{O}$ ratios for succinate were not 
Table 2 Expression level of AtNDA2, AtNDB4, and $\mathrm{NADH}$ :quinone oxidoreductase $(\mathrm{Cl})$ subunits in control cell lines (NDHF) before and after transduction with AtNDA2 and AtNDB4

\begin{tabular}{|c|c|c|c|}
\hline Gene Name & $\begin{array}{l}\text { NDHF-T- } \\
\text { AtNDA2 } \\
\text { (FPKM) }\end{array}$ & $\begin{array}{l}\text { NDHF-T- } \\
\text { AtNDB4 } \\
\text { (FPKM) }\end{array}$ & $\begin{array}{l}\text { NDHF } \\
\text { (FPKM) }\end{array}$ \\
\hline AtNDA2 & 25.29 & n.a. & n.a. \\
\hline AtNDB4 & n.a. & 127.37 & n.a. \\
\hline MT-ND5 & 510.73 & 564.04 & 339.93 \\
\hline NDUFA1 & 92.38 & 109.35 & 84.52 \\
\hline NDUFA10 & 13.45 & 14.93 & 16.52 \\
\hline NDUFA11 & 6.45 & 5.59 & 4.05 \\
\hline NDUFA12 & 45.49 & 57.86 & 65.67 \\
\hline NDUFA13 & 9.52 & 7.51 & 5.60 \\
\hline NDUFA2 & 29.79 & 27.86 & 28.29 \\
\hline NDUFA3 & 34.09 & 30.16 & 26.04 \\
\hline NDUFA4 & 58.43 & 48.98 & 40.88 \\
\hline NDUFA5 & 11.18 & 13.50 & 11.79 \\
\hline NDUFA6 & 31.51 & 37.25 & 42.96 \\
\hline NDUFA7 & 26.23 & 30.32 & 28.79 \\
\hline NDUFA8 & 36.10 & 41.76 & 45.55 \\
\hline NDUFA9 & 32.37 & 39.91 & 50.85 \\
\hline NDUFAB1 & 37.94 & 43.15 & 53.04 \\
\hline NDUFAF1 & 7.62 & 9.04 & 11.40 \\
\hline NDUFAF2 & 24.73 & 37.08 & 39.58 \\
\hline NDUFAF3 & 18.18 & 17.23 & 19.39 \\
\hline NDUFAF4 & 4.53 & 5.80 & 8.58 \\
\hline NDUFAF5 & 2.79 & 3.29 & 5.90 \\
\hline NDUFAF6 & 3.08 & 3.68 & 5.29 \\
\hline NDUFAF7 & 4.29 & 5.73 & 4.65 \\
\hline NDUFB1 & 65.67 & 80.36 & 60.61 \\
\hline NDUFB10 & 40.90 & 42.45 & 45.93 \\
\hline NDUFB11 & 29.52 & 30.82 & 30.67 \\
\hline NDUFB2 & 66.82 & 63.71 & 61.49 \\
\hline NDUFB3 & 45.10 & 56.72 & 58.87 \\
\hline NDUFB4 & 25.68 & 29.74 & 27.33 \\
\hline NDUFB5 & 36.56 & 41.54 & 46.47 \\
\hline NDUFB6 & 33.60 & 40.03 & 40.62 \\
\hline NDUFB7 & 41.06 & 37.93 & 29.89 \\
\hline NDUFB8 & 30.74 & 38.31 & 33.31 \\
\hline NDUFB9 & 27.26 & 31.96 & 36.70 \\
\hline NDUFC1 & 18.28 & 20.27 & 17.64 \\
\hline NDUFC2 & 9.53 & 10.69 & 11.96 \\
\hline NDUFS1 & 22.29 & 27.30 & 42.39 \\
\hline NDUFS2 & 43.52 & 51.22 & 54.07 \\
\hline NDUFS3 & 18.07 & 22.73 & 25.74 \\
\hline NDUFS4 & 59.89 & 66.07 & 70.66 \\
\hline NDUFS5 & 157.96 & 182.22 & 171.86 \\
\hline NDUFS6 & 36.55 & 37.20 & 42.90 \\
\hline NDUFS7 & 3.28 & 2.66 & 2.09 \\
\hline NDUFS8 & 27.07 & 24.71 & 27.89 \\
\hline NDUFV1 & 37.28 & 37.51 & 38.60 \\
\hline NDUFV2 & 65.14 & 91.45 & 93.46 \\
\hline NDUFV3 & 9.58 & 9.60 & 12.39 \\
\hline $\begin{array}{ll}\text { Median } & \text { CI } \\
\text { FPKM } & \\
\end{array}$ & 30.26 & 34.52 & 35.01 \\
\hline
\end{tabular}

Expression levels are indicated in FPKM. In gray are labelled the $\mathrm{NADH}$ :quinone oxidoreductase subunits, in green the two NDH-2 genes. Median FPKM for $\mathrm{Cl}$ subunits in both cell lines is given in red significantly lower in transfected cells, $0.43 \pm 0.05$ and $0.39 \pm 0.02$, respectively, we observed a reduction of respiratory control index in transfected cells (Fig. 5). This is likely due to the metabolic conversion of a fraction of succinate to glutamate, which later enters the oxidation machinery proceeding through $\mathrm{CI}$ and AtNDA2.

When ATP instead of ADP was used, we observed only a very low OXPHOS stimulation in both transfected and untransfected cell lines, providing an additional confirmation that our measurments were not significantly affected by ATPase mediated ATP recycling to ADP (not shown).

A further evidence of the likely competition between CI and AtNDA2, came from calculation of $K_{M}$ for $\mathrm{NADH}$ in both transfected and untransfected cell lines (Fig. 6). CI affinity for NADH was estimated in untransfected control cells considering only rotenone sensitive internal NADH:quinone oxidoreductase activity, while, to evaluate AtNDA2 affinity for $\mathrm{NADH}$, we exclusively analyzed rotenone insensitive activity in AtNDA2transfected control cells. Our estimation of $\mathrm{CI}$ and AtNDA2 $K_{\mathrm{M}}$ gave values of $2.7 \pm 0.4 \mu \mathrm{M}$ and $9.7 \pm$ $3.3 \mu \mathrm{M}$, respectively. Hence, when transfected in human cells, $K_{M}$ of AtNDA2 for NADH appear to be about only 3 folds higher than $\mathrm{K}_{\mathrm{M}}$ of $\mathrm{CI}$ for $\mathrm{NADH}$, i.e. in the same order of magnitude; this gap is probably insufficient to prevent competition for the substrate within physiological range of NADH concentration inside the mitochondria, therefore indirectly confirming that upon these experimental conditions, it seems likely that $\mathrm{CI}$ and AtNDA2 compete for the oxidation of NADH.

\section{Discussion}

$\mathrm{CI}$ is the largest complex of the respiratory chain, consisting of 44 different subunits encoded by both nDNA and mtDNA. These subunits are assembled in a precise order by numerous assembly factors [1]. Thus, pathogenic mutations in genes encoding either for structural subunits or for assembly factors, can result in the enzymatic impairment of $\mathrm{CI}$, often with a yet poorly understood tissue-specificity and time-dependency. Besides these mechanisms, CI deficiency may arise as a consequence of mutations in genes encoding proteins involved in mitochondrial translation, in iron-sulphur cluster assembly, and mtDNA-depletion associated genes $[31,32]$. This could explain why CI deficiency is the far most common finding in mitochondrial disorders.

In terms of therapeutic approach, having a unique treatment applicable to all CI deficiencies, regardless of the genetic cause, would be desirable. A bypassing strategy using alternative dehydrogenase proteins seems to offer such possibility. Indeed the monomeric NADH dehydrogenase of yeast, ScNDI1, inserted in CI-deficient cells by Yagi and colleagues [14], presented an apparent beneficial effect on several experimental models in vitro 


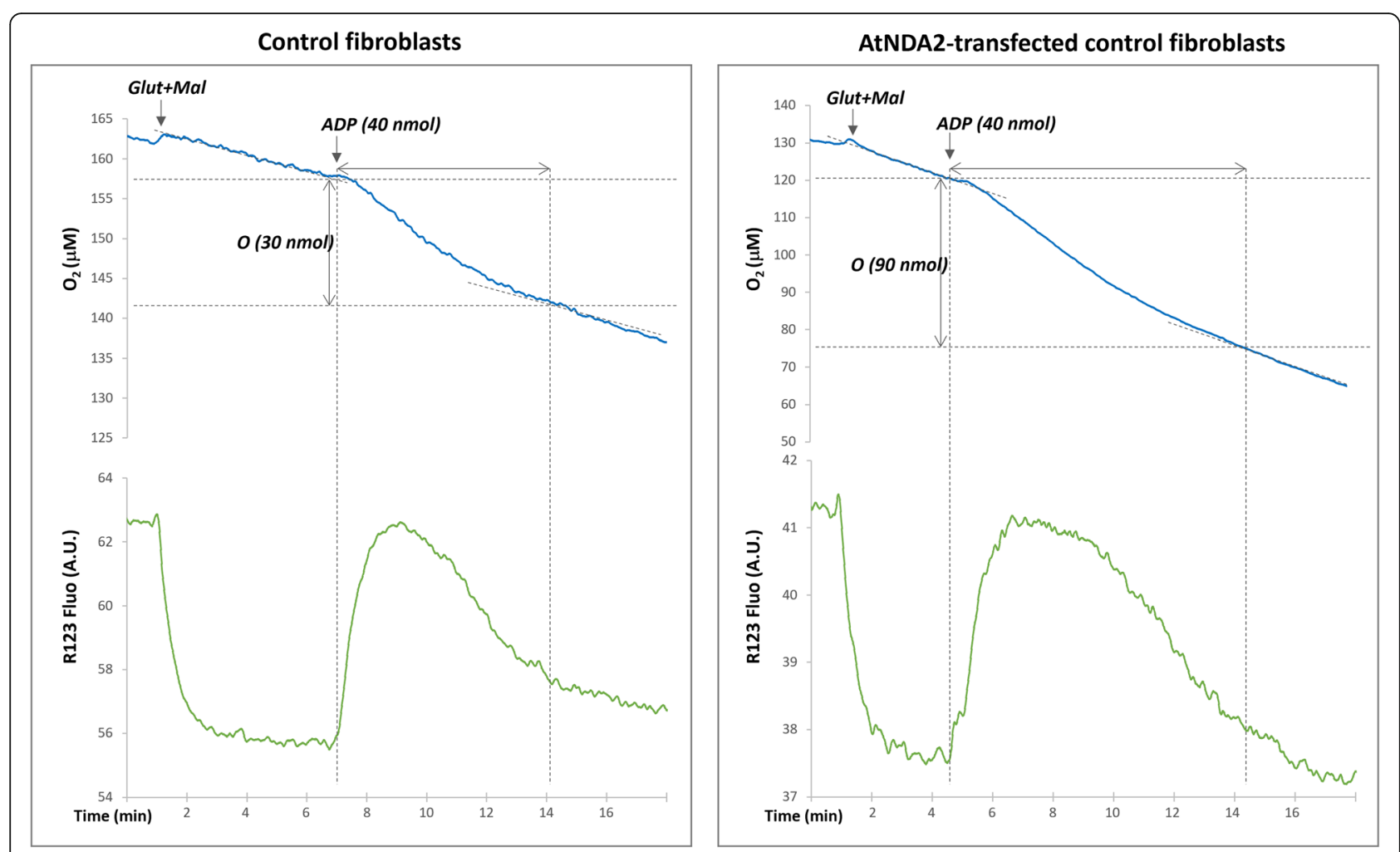

Fig. 4 Evaluation of mitochondrial respiration: Mitochondrial membrane potential variations assessed by rhodamine 123 fluorescence and oxygen uptake measured with optode device in digitonine permeabilized fibroblasts (representative graphs for the control fibroblasts - left panel and AtNDA2-transfected control fibroblasts - right panel). The reaction was started by the addition of glutamate/malate, followed by injections of ADP (see text). Note that the amount of oxygen reduced during ADP phosphorylation is significantly higher in AtNDA2 transfected cells comparing to control

and in vivo [8-13]. Nevertheless, it lowered $\mathrm{P} / \mathrm{O}$ values for CI-dependent oxidation of NADH indicating reduced ATP synthesis upon ScNDI1 transfection in control mammalian cells [14] raising questions about its feasibility as treatment for patients with impaired but residual CI activity, where prevalence of ScNDI1 over residual CI activity could worsen metabolic disturbances and lower OXPHOS energetic yield. The consequences on cellular homeostasis could be potentially deleterious, since ATP synthesis decline is one of the major pathomechanism involved in the CI deficiency-related phenotype.

A. thaliana NDH-2 naturally coexist with CI: their activity is stimulated when electron flux through mitochondrial OXPHOS is slowed down, most likely depending on their intrinsic enzymatic properties [15-18, 33]. Hence, they represent valuable candidates for complementing a defective $\mathrm{CI}$ activity without competing with it. AtNDA2, and AtNDB4, in particular, show a substrate preference for NADH over NADPH and their catalytic activity is $\mathrm{Ca}^{2+}$ independent, similarly to $\mathrm{CI}$. AtNDA2 is typically detected in the mitochondrial inner membrane, facing the matrix $[15,29]$, but there is evidence of an additional peroxisomal location [34]. AtNDB4 instead faces the intermembrane space. To target these proteins specifically to the mitochondrial matrix of mammalian fibroblasts, the plant-specific mitochondrial targeting sequence (MTS) was replaced with the human MTS.

AtNDA2, and AtNDB4 were both able to rescue the biochemical defect when expressed in CI-deficient cells, as indicated by increased respiration determined by oxygen consumption determination and lowered SOD activity, a surrogate of ROS production. Functional expression of plant NDH-2 was further indicated by complementation of defective $\mathrm{CI}$ on spectrophotometric assays, since both enzymes were able to re-establish NADH:quinone oxidoreductase activity close to control values. Besides, they did not affect cell growth both in standard culture conditions and in the case of glucose deprivation, when cells are forced to switch on OXPHOS for energy production. This observation suggested a lack of competition with $\mathrm{CI}$ in standard culture conditions. However, the absence of any apparent effect on cellular growth could be also due to inadequate strength of competition or low level of NDA2 in proportion to CI preventing from detection of such a competition.

We decided to focus on AtNDA2, which represents the most promising candidate to replace $\mathrm{CI}$ in deficient cells based on its location and activity profile within 


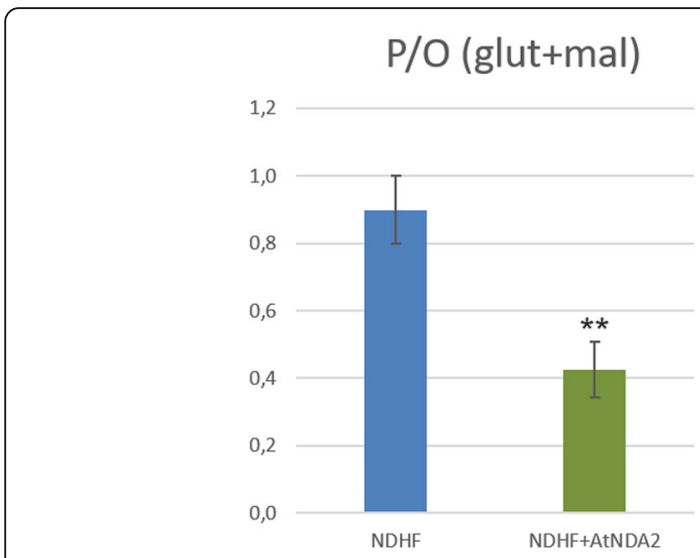

A

RC (glut+mal)

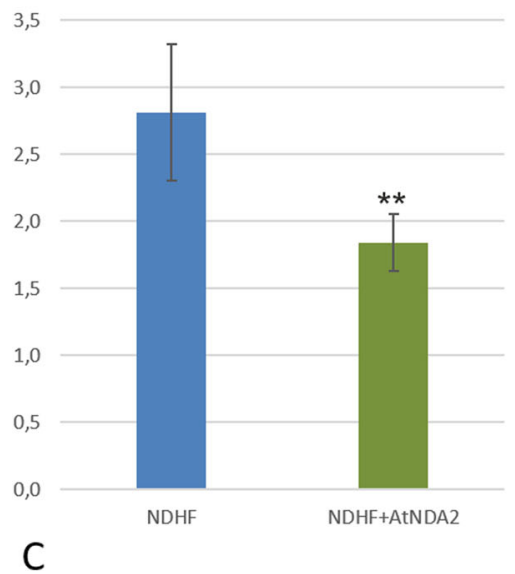

\section{$\mathrm{P} / \mathrm{O}$ (succinate)}

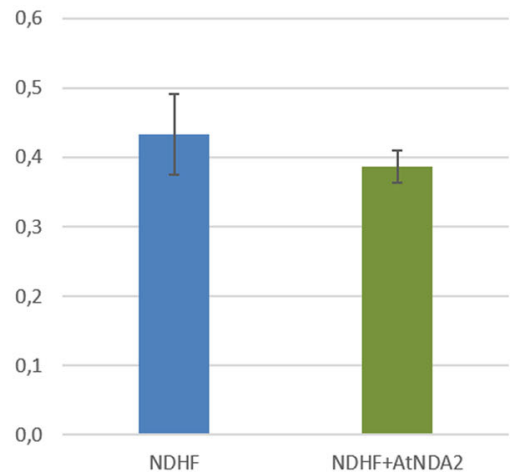

B

\section{$\mathrm{RC}$ (succinate)}

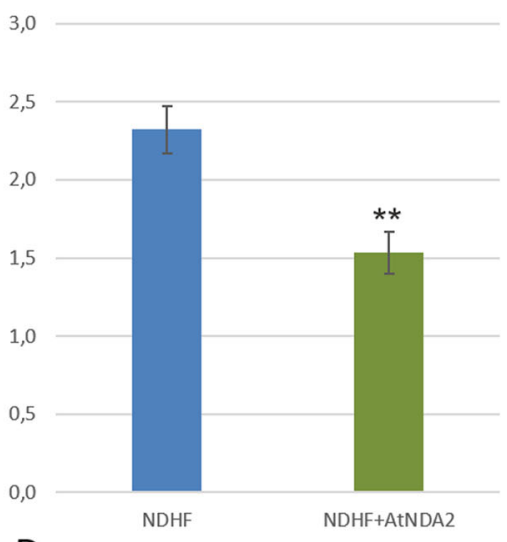

D

Fig. 5 RC (respiratory control) and P/O coupling ratios: Comparison of P/O values (a and $\mathbf{b}$ ) and Respiratory Control Index (c and $\mathbf{d}$ ) with Glutamate/Malate $(\mathbf{a}, \mathbf{c})$ and Succinate $(\mathbf{b}, \mathbf{d})$ in non-transfected control and AtNDA2 transfected control cells

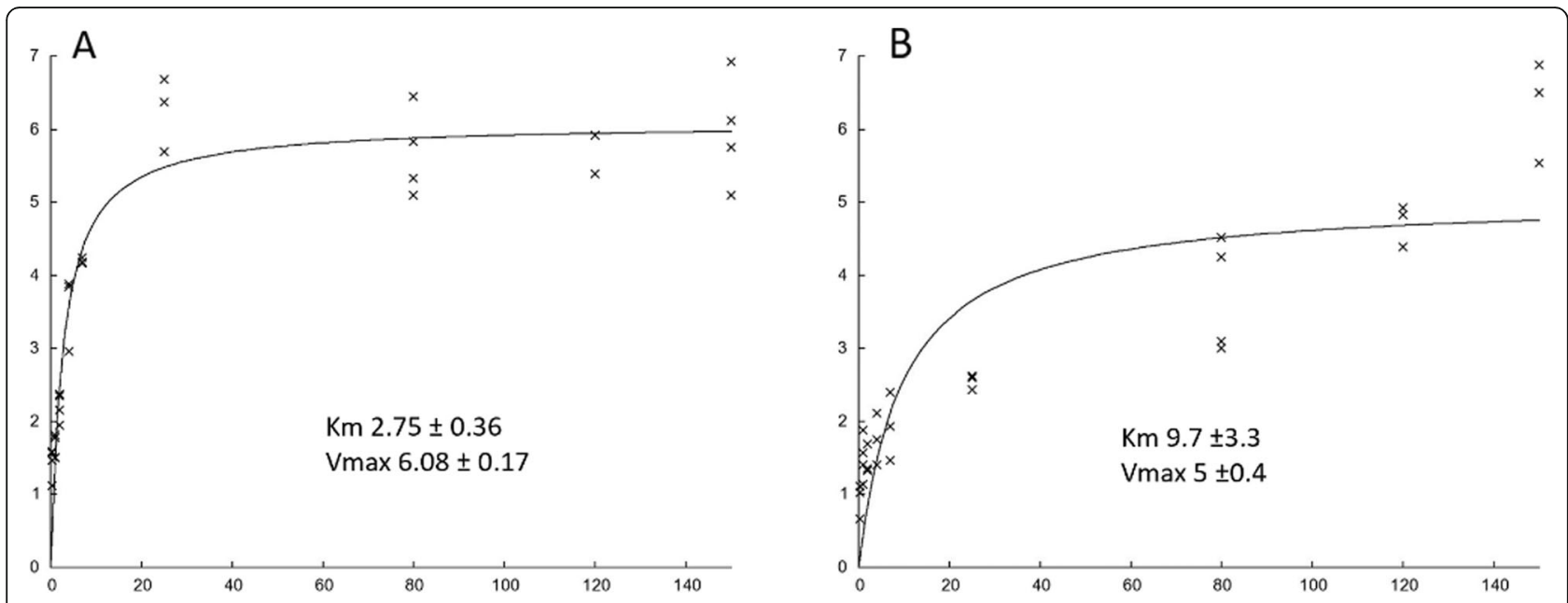

Fig. $6 K_{M}$ evaluation: Plots of NADH:quinone oxidoreductase activity $(y)$ as a function of NADH concentration $(\mu M)(x)$ : a rotenone-sensitive NADH:quinone oxidoreductase activity in control cells; $\mathbf{b}$ rotenone-resistant NADH:quinone oxidoreductase activity in AtNDA2 transfected control cells 
plant mitochondria $[16,18,35]$ To verify this possibility, we expressed AtNDA2 in human control fibroblasts and evaluated the effect on the ADP phosphorylation levels under different respiratory substrates. Using an NADH related substrate (glutamate/malate), the $\mathrm{P} / \mathrm{O}$ ratio of transfected cells was lowered by half when compared to control cells. This indicates that AtNDA2 is active when expressed in control cells and competes with CI for electron transfer from NADH to quinone. Additionally, rotenone resistant NADH oxidase activity in AtNDA2control cells has an apparent $K_{M}$ of $9.7 \mu \mathrm{M}$ for NADH which is slightly over 3-fold higher than affinity of $\mathrm{CI}$ for NADH evaluated in control cells $(2.7 \mu \mathrm{M})$. Hence, in our experimental model AtNDA2 and CI affinity for the same substrate seem to fall within a similar order of magnitude, thus supporting the existence of a competition in human cells.

However, there are few important limitations linked to this measurement that need to be considered.

Previous studies on plant mitochondria had calculated rotenone-resistant NADH oxidase activity of the inner membrane to be up to 10 -fold higher than $\mathrm{K}_{M}$ of CI $[17,29,36]$, although other authors later reported a considerably lower value of $13.9 \mu \mathrm{M}$ [37], which is closer to our results.

Likewise, reported apparent $K_{M}$ values of $C I$ for $\mathrm{NADH}$ are quite heterogeneous, ranging from $2 \mu \mathrm{M}$ to $20 \mu \mathrm{M}[38-41]$.

There are few important considerations to explain the observed intergroup variability. First of all, the development of a specific method to evaluate kinetic properties of $\mathrm{CI}$ has notoriously been a recurrent challenge for researchers [23, 42, 43].

Besides this, we should also consider methodological heterogeneity (e. g. sample preparation, category of quinone-analogues employed as electron acceptors, difficulty to accurately estimate enzymatic activity when dealing with extremely low concentration of the substrate etc). Indeed, kinetic properties of these enzymes have been mainly estimated on isolated mitochondria/ submitochondrial preparations and on different cell lineages, while we studied permeabilised cell preparations, which are unavoidably contaminated to some extent by soluble NADH dehydrogenases activities. Moreover, AtNDA2 compartmentation within the inner surface of mitochondrial membrane or its association to a supramolecular complex (enzyme malic/specific quinone pool/AOXs) under native conditions, could contribute to its distinctive kinetic properties for NADH and to prevent competition with $\mathrm{CI}$, thus ensuring AtNDA2 activity only in specific physiological circumstances [44].

Most reasonably, the apparent competition with the endogenous OXPHOS system could depend on the plant enzyme concentration within human mitochondria. In our experimental system we used a strong promoter and reached an overall AtNDA2 RNA expression level (25 FPKM) falling in the range of complex I subunit expression (median 29 FPKM). Interpreting these data as a rough measure to approximate the protein levels (unfortunately, we lack information about the post-transcriptional effects for both, AtNDA2 and complex I), would indicate quite high AtNDA2 levels when compared to A. thaliana, where AtNDA2 expression has been reported to be up to 10-fold lower than complex I [45]. This very high level of plant enzyme could thus result in the observed competition between AtNDA2 and complex I in our cellular model.

Therefore, our data suggest to test experimental systems exhibiting lower expression levels of NDH-2 for further studies.

\section{Conclusions}

In conclusion, we showed that transfection of plant $\mathrm{NDH}-2$ was able to rescue $\mathrm{CI}$ defect in vitro. However, AtNDA2, the most promising candidate based on its properties in plants, exhibits competing activity with human CI when expressed at high levels, thus raising concerns which need to be considered in case of its application to human therapy. In cells with substantial CI activity, a balance in terms of energy production and metabolic dysfunctions needs to be determined, in which the gain of additional NADH oxidation is more beneficial than the reduced ATP production through competition with CI. If not controlled, consequences of this uncoupling effect are unpredictable in vivo, and risk to be deleterious in affected patients. A substantial amount of translational work still has to be done in the near future, from genetic manipulation of the transfected plant product to possible modification of its enzymatic properties, to the generation of an animal model to test its effects in vivo.

Nevertheless, we have moved an important step towards a deeper comprehension of potential advantages and drawbacks of trans-kingdom replacing therapy for respiratory chain defects.

\section{Additional file}

Additional file 1: Supplementary methods. Supplementary results.
Table S1. Expression level of AtNDA2, AtNDB4, and NADH:quinone
oxidoreductase $(\mathrm{Cl})$ subunits in various compartment of A.thaliana. (DOCX
$105 \mathrm{~kb}$ )

Acknowledgements

We thank Dr. Dejana Mokranjac for providing genomic DNA of the yeast, and Prof. Takao Yagi for providing the antibody against the yeast NDI1. The authors declare no conflicts of interest. 


\section{Authors' contributions}

AC performed ADP/O essays and evaluation of $\mathrm{K}_{\mathrm{M}}$, analyzed data and wrote the manuscript. Al generated all transgenic constructs, performed transduction experiment and Western Blotting, analyzed Seahorse data and wrote the manuscript. CT cultured cells and isolated the RNA. LSK performed Seahorse experiments. TS processed and analyzed the RNA-seg data. JB, MP and PB performed spectrophotometry and SOD experiments. AGR provided expertise regarding Western blot experiments. VT, PR, MR, HP and MS designed the experiments and wrote the manuscript. All authors read and approved the final manuscript.

\section{Author's information}

Not applicable.

\section{Funding}

This study was supported by the German BMBF and Horizon2020 through E-Rare project GENOMIT (01GM1603 and 01GM1906B to H.P.); Horizon2020 Project SOUND (633974 to H.P.); German Network for Mitochondria Disorders (mitoNET 01GM1113C to H.P.); AMMi (Association contre les Maladies Mitochondriales) grants to M.P., P.R., P.B. and M.S.; E-Rare project GENOMIT (ANR-15-RAR3-0012 to P.R., A.C. and M.S.); Italian Ministry of Health (RF-201602361495 to A.C.); Mitocon (Grant no. 2018-01 to V.T.); Mariani Foundation (V.T.).

\section{Availability of data and materials}

The datasets used and/or analysed during the current study are available from the corresponding author on reasonable request.

\section{Ethics approval and consent to participate}

All procedures performed in studies involving human participants were in accordance with the ethical standards of the institutional and/or national research committee and with the 1964 Helsinki declaration and its later amendments or comparable ethical standards.

Informed consent was obtained from all individual participants (or their legal tutors) included in the study prior to performing cutaneous biopsies.

\section{Consent for publication}

Not applicable.

\section{Competing interests}

The authors declare that they have no competing interests.

\section{Author details}

'UMR1 141, PROTECT, INSERM, Université de Paris, Paris, France. ${ }^{2}$ Unit of Medical Genetics and Neurogenetics, Fondazione IRCCS Istituto Neurologico Carlo Besta, Milan, Italy. ${ }^{3}$ Institute of Human Genetics, Helmholtz Zentrum München, German Research Center for Environmental Health, Neuherberg, Germany. Institute of Human Genetics, Technische Universität München, Munich, Germany. ${ }^{5}$ Reference Center for Inborn Errors of Metabolism, Hôpital Universitaire Robert Debré, APHP, Paris, France. ${ }^{6}$ Department of Biology, Lund University, Biology building A, Sölvegatan 35, SE-22362 Lund, Sweden. ${ }^{7}$ Dr. von Hauner Children's Hospital, Department of Pediatrics, University Hospital, Ludwig-Maximilians-Universität (LMU), Munich, Germany.

Received: 14 June 2019 Accepted: 30 August 2019

Published online: 29 October 2019

\section{References}

1. Sánchez-Caballero L, Guerrero-Castillo S, Nijtmans L. Unraveling the complexity of mitochondrial complex I assembly: a dynamic process. Biochim Biophys Acta. 2016;1857(7):980-90. https://doi.org/10.1016/j.bbabio. 2016.03.031

2. Ripple $\mathrm{MO}$, Kim N, Springett R. Mammalian complex I pumps 4 protons per 2 electrons at high and physiological proton motive force in living cells. J Biol Chem. 2013;288(8):5374-80.

3. Brandt U. Energy converting NADH:quinone oxidoreductase (complex I). Annu Rev Biochem. 2006;75:69-92.

4. Haack TB, Haberberger B, Frisch EM, Wieland T, luso A, Gorza M, et al. Molecular diagnosis in mitochondrial complex I deficiency using exome sequencing. J Med Genet. 2012;49(4):277-83. https://doi.org/10.1136/ jmedgenet-2012-100846.
5. Saari S, Garcia GS, Bremer K, Chioda MM, Andjelković A, Debes PV, et al. Alternative respiratory chain enzymes: therapeutic potential and possible pitfalls. Biochim Biophys Acta Mol Basis Dis. 2019;1865(4):854-66. https://doi. org/10.1016/j.bbadis.2018.10.012

6. Cannino G, El-Khoury R, Pirinen M, Hutz B, Rustin P, Jacobs HT, et al. Glucose modulates respiratory complex I activity in response to acute mitochondrial dysfunction. J Biol Chem. 2012;287(46):38729-40. https://doi.org/10.1074/jbc. M112.386060.

7. McDonald AE, Gospodaryov DV. Alternative $\mathrm{NAD}(\mathrm{P}) \mathrm{H}$ dehydrogenase and alternative oxidase: proposed physiological roles in animals. Mitochondrion. 2019;45:7-17. https://doi.org/10.1016/j.mito.2018.01.009.

8. Bai Y, Hajek P, Chomyn A, Chan E, Seo BB, Matsuno-Yagi A, et al. Lack of complex I activity in human cells carrying a mutation in MtDNA-encoded ND4 subunit is corrected by the Saccharomyces cerevisiae NADH-quinone oxidoreductase (NDI1) gene. J Biol Chem. 2001;276(42):38808-13.

9. Bai Y, Hu P, Park JS, Deng JH, Song X, Chomyn A, et al. Genetic and functional analysis of mitochondrial DNA-encoded complex I genes. Ann N Y Acad Sci. 2004;1011:272-83.

10. Cho J, Hur JH, Graniel J, Benzer S, Walker DW. Expression of yeast NDI1 rescues a Drosophila complex I assembly defect. PLoS One. 2012;7(11): e50644. https://doi.org/10.1371/journal.pone.0050644.

11. Marella M, Seo BB, Thomas BB, Matsuno-Yagi A, Yagi T. Successful amelioration of mitochondrial optic neuropathy using the yeast NDI1 gene in a rat animal model. PLoS One. 2010;5(7):e11472.

12. Marella M, Seo BB, Flotte TR, Matsuno-Yagi A, Yagi T. No immune responses by the expression of the yeast Ndi1 protein in rats. PLoS One. 2011;6(10):e25910.

13. Chadderton N, Palfi A, Millington-Ward S, Gobbo O, Overlack N, Carrigan M, et al. Intravitreal delivery of AAV-NDI1 provides functional benefit in a murine model of Leber hereditary optic neuropathy. Eur J Hum Genet. 2013;21(1):62-8.

14. Barber-Singh J, Seo BB, Matsuno-Yagi A, Yagi T. Protective role of rAAVNDI1, serotype 5, in an acute MPTP mouse Parkinson's model. Parkinsons Dis. 2010;2011:438370. https://doi.org/10.4061/2011/438370.

15. Seo BB, Matsuno-Yagi A, Yagi T. Modulation of oxidative phosphorylation of human kidney 293 cells by transfection with the internal rotenoneinsensitive NADH-quinone oxidoreductase (NDI1) gene of Saccharomyces cerevisiae. Biochim Biophys Acta. 1999:1412(1):56-65.

16. Michalecka AM, Svensson AS, Johansson Fl, Agius SC, Johanson U, Brennicke $A$, et al. Arabidopsis genes encoding mitochondrial type II NAD(P) H dehydrogenases have different evolutionary origin and show distinct responses to light. Plant Physiol. 2003;133(2):642-52.

17. Rasmusson $A G$, Soole $\mathrm{KL}$, Elthon TE. Alternative $\mathrm{NAD}(\mathrm{P}) \mathrm{H}$ dehydrogenases of plant mitochondria. Annu Rev Plant Biol. 2004;55:23-39.

18. Moller IM, Palmer JM. Direct evidence for the presence of a rotenoneresistant $\mathrm{NADH}$ dehydrogenase on the inner surface of the inner membrane of plant mitochondria. Physiol Plant. 1982;54(3):267-74.

19. Rasmusson AG, Geisler DA, Møller IM. The multiplicity of dehydrogenases in the electron transport chain of plant mitochondria. Mitochondrion. 2008; 8(1):47-60 Review

20. El-Khoury R, Kemppainen KK, Dufour E, Szibor M, Jacobs HT, Rustin P. Engineering the alternative oxidase gene to better understand and counteract mitochondrial defects: state of the art and perspectives. $\mathrm{Br}$ J Pharmacol. 2014;171(8):2243-9. https://doi.org/10.1111/bph.12570.

21. Anderson SL, Chung WK, Frezzo J, Papp JC, Ekstein J, DiMauro S, et al. A novel mutation in NDUFS4 causes Leigh syndrome in an Ashkenazi Jewish family. J Inherit Metab Dis. 2008;31(Suppl 2):S461-7. https://doi.org/10.1007/ s10545-008-1049-9.

22. Kremer LS, Prokisch $\mathrm{H}$. Identification of disease-causing mutations by functional complementation of patient-derived fibroblast cell lines. Methods Mol Biol. 2017;1567:391-406. https://doi.org/10.1007/978-1-4939-6824-4_24.

23. Bénit P, Goncalves S, Philippe Dassa E, Brière JJ, Martin G, Rustin P. Three spectrophotometric assays for the measurement of the five respiratory chain complexes in minuscule biological samples. Clin Chim Acta. 2006; 374(1-2):81-6.

24. Chretien D, Benit P, Chol M, Lebon S, Rötig A, Munnich A, et al. Assay of mitochondrial respiratory chain complex I in human lymphocytes and cultured skin fibroblasts. Biochem Biophys Res Commun. 2003;301:222-4.

25. Bradford MM. A rapid and sensitive method for the quantitation of microgram quantities of protein utilizing the principle of protein-dye binding. Anal Biochem. 1976;72:248-54.

26. Geromel V, Kadhom N, Cebalos-Picot I, Ouari O, Polidori A, Munnich A et al. Superoxide-induced massive apoptosis in cultured skin fibroblasts harboring 
the neurogenic ataxia retinitis pigmentosa (NARP) mutation in the ATPase-6 gene of the mitochondrial DNA. Hum Mol Genet. 2001;10(11):1221-8.49.

27. Kremer LS, Bader DM, Mertes C, Kopajtich R, Pichler G, luso A, et al. Genetic diagnosis of Mendelian disorders via RNA sequencing. Nat Commun. 2017;8: 15824. https://doi.org/10.1038/ncomms15824.

28. Breuer ME, Willems PH, Smeitink JA, Koopman WJ, Nooteboom M. Cellular and animal models for mitochondrial complex I deficiency: a focus on the NDUFS4 subunit. IUBMB Life. 2013;65(3):202-8. https://doi. org/10.1002/iub.1127.

29. Geisler DA, Broselid C, Hederstedt L, Rasmusson AG. Ca2+-binding and $\mathrm{Ca} 2+-$ independent respiratory $\mathrm{NADH}$ and NADPH dehydrogenases of Arabidopsis thaliana. J Biol Chem. 2007;282(39):28455-64.

30. Hinkle PC. P/O ratios of mitochondrial oxidative phosphorylation. Biochim Biophys Acta. 2005;1706(1-2):1-11.

31. Scheffler IE. Mitochondrial disease associated with complex I (NADH-CoO oxidoreductase) deficiency. J Inherit Metab Dis. 2015;38(3):405-15. https:// doi.org/10.1007/s10545-014-9768-6.

32. Emmanuele V, López LC, Berardo A, Naini A, Tadesse S, Wen B, et al. Heterogeneity of coenzyme Q10 deficiency: patient study and literature review. Arch Neurol. 2012;69(8):978-83. https://doi.org/10.1001/archneurol. 2012.206 Review. Erratum in: Arch Neurol. 2012 Jul;69(7):886.

33. Lee $\mathrm{CP}$, Eubel $\mathrm{H}$, Millar $\mathrm{AH}$. Diurnal changes in mitochondrial function reveal daily optimization of light and dark respiratory metabolism in Arabidopsis. Mol Cell Proteomics. 2010;9(10):2125-39. https://doi.org/10. 1074/mcp.M110.001214.

34. Carrie C, Murcha MW, Kuehn K, Duncan O, Barthet M, Smith PM, et al. Type II NAD(P) H dehydrogenases are targeted to mitochondria and chloroplasts or peroxisomes in Arabidopsis thaliana. FEBS Lett. 2008;582(20):3073-9. https://doi.org/10.1016/j.febslet.2008.07.061.

35. Elhafez D, Murcha MW, Clifton R, Soole KL, Day DA, Whelan J. Characterization of mitochondrial alternative $N A D(P) H$ dehydrogenases in Arabidopsis: intraorganelle location and expression. Plant Cell Physiol. 2006; 47(1):43-54.

36. Soole KL, Dry IB, James AT, Wiskich JT. The kinetics of NADH oxidation by complex I of plant mitochondria. Physiol Plant. 1990;80:75-82.

37. Rasmusson AG, Møller IM. NAD(P) $H$ dehydrogenases on the inner surface of the inner mitochondrial membrane studied using inside-out particles. Physiol Plant. 1991;83:357-65.

38. Hatefi Y, Stiggal DL. In: Boyer PD, editor. The enzymes. 3rd ed. New York: Academic; 1976. p. 175-297.

39. Vinogradov AD. Kinetics, control, and mechanism of ubiquinone reduction by the mammalian respiratory chain-linked NADH-ubiquinone reductase. Bioenerg Biomembr. 1993;25(4):367-75.

40. Majander A, Huoponen K, Savontaus ML, Nikoskelainen E, Wikström M. Electron transfer properties of NADH:ubiquinone reductase in the ND1/3460 and the ND4/11778 mutations of the Leber hereditary optic neuroretinopathy (LHON). FEBS Lett. 1991;292(1-2):289-92.

41. Barrientos A, Kenyon L, Moraes CT. Human xenomitochondrial cybrids. Cellular models of mitochondrial complex I deficiency. J Biol Chem. 1998; 273(23):14210-7.

42. de Wit LE, Scholte HR, Sluiter W. Correct assay of complex I activity in human skin fibroblasts by timely addition of rotenone. Clin Chem. 2008; 54(11):1921-2; author reply 1922-4. https://doi.org/10.1373/clinchem.2008. 104802.

43. Yano T. The energy-transducing NADH: quinone oxidoreductase, complex I. Mol Aspects Med. 2002;23(5):345-68.

44. Rustin P, Moreau F. Malic enzyme activity and cyanide-insensitive electron transport in plant mitochondria. Biochem Biophys Res Commun. 1979;88(3): 1125-31.

45. Klodmann J, Sunderhaus S, Nimtz M, Jänsch L, Braun HP. Internal architecture of mitochondrial complex I from Arabidopsis thaliana. Plant Cell. 2010;22(3):797-810. https://doi.org/10.1105/tpc.109.073726.

\section{Publisher's Note}

Springer Nature remains neutral with regard to jurisdictional claims in published maps and institutional affiliations.

\section{Ready to submit your research? Choose BMC and benefit from}

- fast, convenient online submission

- thorough peer review by experienced researchers in your field

- rapid publication on acceptance

- support for research data, including large and complex data types

- gold Open Access which fosters wider collaboration and increased citations

- maximum visibility for your research: over $100 \mathrm{M}$ website views per year

At BMC, research is always in progress.

Learn more biomedcentral.com/submissions 